\title{
Diseños epidemiológicos y dinámica de producción científica en los trabajos de grado de una facultad de odontología colombiana
}

\author{
Designs of epidemiological studies and dynamics of scientific production in \\ academic dissertations at a Colombian school of dentistry
}

\author{
Martha Juliana Rodríguez ${ }^{(D)}$, Juan José Sánchez ${ }^{2}$ (D), Sonia Constanza Concha-Sánchez ${ }^{3}$ (D) \\ Citación: Rodríguez MJ, Sánchez JJ, Concha-Sánchez SC. Diseños epidemiológicos y dinámica de producción científica en los trabajos de grado \\ de una facultad de odontología colombiana. Ustasalud 2021; 20: 21-30.
}

Licencia Creative Commons

\section{(c) (1) () $\Theta$}

La revista Ustasalud declara que su contenido se rige bajo la licencia Creative Commons de Atribución - No comercial - Sin Derivar 4.0 Internacional. Por 作 alterarlos, adicionalmente se debe reconocer la autoría de las personas que figuran en las publicaciones, pero estas no podrán ser comercializadas.

\section{Resumen}

Determinar el diseño de estudio epidemiológico y la dinámica de producción científica en los trabajos de grado presentados como requisito de grado de los programas de pregrado y posgrado de la Facultad de Odontología de la Universidad Santo Tomás Seccional Bucaramanga.

Se realizó un estudio observacional descriptivo de corte transversal con la totalidad de los trabajos de grado de la Facultad de Odontología publicados en el Repositorio Institucional. Se incluyó la información proveniente del "registro completo del ítem" de los 479 trabajos que no estuvieran repetidos y que su fecha de publicación fuera posterior a 2014. Se analizaron las siguientes variables: disponibilidad en texto completo, número de estudiantes, nombre del director, nivel de escolaridad de este, número de codirectores, nivel de escolaridad del primer codirector, año de publicación, programa académico, tipo de estudio y divulgación en revistas científicas. Se calcularon frecuencias y proporciones para las variables cualitativas, y medidas de tendencia central y dispersión para las cuantitativas.

Se evidenció que $338(71,0 \%)$ trabajos de grado fueron publicados por pregrado y $360(75,6 \%)$ estaban disponibles en texto completo. Se encontró que $252(52,9 \%)$ correspondían a estudios observacionales; de estos, $237(94,1 \%)$ eran de corte transversal. Más de la mitad de los trabajos de grado fueron dirigidos por docentes con maestría. Trece $(2,7 \%)$ trabajos de grado fueron publicados en nueve revistas científicas, de las cuales seis eran colombianas.

El diseño epidemiológico más utilizado en los trabajos de grado fue el observacional de corte transversal al verse reflejado en $237(94,1 \%)$ documentos.

Palabras clave: Estudio observacional, estudios transversales, tesis académica, facultades de odontología, estudiantes.

\begin{abstract}
To determine epidemiological study design and the dynamics of scientific production in academic dissertations presented as a requirement for the undergraduate and graduate programs at the School of Dentistry in Universidad Santo Tomás Seccional Bucaramanga.

A descriptive, cross-sectional observational study was conducted with all academic dissertations from the School of Dentistry published in the Institutional Repository. The information included was taken from the "complete item record" of the 479 documents that were not duplicated and whose publication date was after 2014. The following variables were analyzed: availability in full text, number of students, project's director name, his/her level of education, number of co-directors, first co-director level of education, year of publication, academic program, type of study, and whether the dissertation had resulted in a scientific publication. Frequencies and proportions were calculated for qualitative variables and measures of central tendency and dispersion for quantitative ones.
\end{abstract}

It was observed that $338(71.0 \%)$ academic dissertations were published by undergraduate students, and $360(75.6 \%)$ were available in full text. It was found that $252(52.9 \%)$ were observational studies. Of these, 237 $(94.1 \%)$ were cross-sectional. More than half of the project tutors presented a master's degree. Thirteen (2.7\%) academic dissertations were published in nine scientific journals, six of which were Colombian.

It was identified that $237(94,1 \%)$ academic dissertations had a cross-sectional study design.

Keywords: Observational study, cross-sectional studies, academic dissertation, dental schools, students.
1 Magíster en Epidemiología, Universidad Industrial de Santander, docente Facultad de Odontología, Universidad Santo Tomás.

2 Estudiante Facultad de Odontología, Universidad Santo Tomás.

3 Doctora en Salud Pública, Universidad Nacional de Colombia, docente Facultad de Odontología, Universidad Santo Tomás.

Autor de correspondencia: Martha Juliana Rodríguez Correo electrónico: marthajuro@gmail.com
Recibido para publicación:
22 de enero de 2021
Aceptado para publicación:
5 de abril de 2021 


\section{INTRODUCCIÓN}

El diseño en un proyecto de investigación es un aspecto fundamental que determina la dinámica y los criterios que pueden ser evaluados en el proceso investigativo. Thiese refiere que los estudios, generalmente, se catalogan como primarios (datos originalmente recolectados para un propósito investigativo) o secundarios (datos provenientes de investigaciones primarias). A su vez, los estudios primarios se clasifican según la temporalidad (prospectivo o retrospectivo), el uso de los resultados (básicos o aplicados), el propósito de la investigación (descriptivo o analítico), la aplicación de los resultados (preventivo, diagnóstico o de tratamiento) o de acuerdo con el papel que juega el investigador (observacional o de intervención). Es así como los estudios observacionales se refieren a aquellos diseños epidemiológicos en los que el investigador no asigna la exposición, en lugar de ello "observa" la relación natural entre la exposición y el desenlace [1].

Entre los estudios observacionales se destacan los de corte transversal que se caracterizan por recoger la información en un momento del tiempo, es decir, no existe una dimensión temporal $[2,3]$. Esta situación implica que los datos sobre la exposición y la presencia del evento se recogen en un único instante [4]. Por tal motivo, no es posible considerar este tipo de diseño si se desea identificar la causa o etiología de un evento [5], aunque una excepción podría ser cuando se estudia una exposición inmutable o que no cambia a lo largo del tiempo como el sexo o el tipo sanguíneo [6].

Rothman y Lash señalan que cuando un estudio de corte transversal se realiza con el objetivo de establecer la prevalencia de un evento, recibe el nombre de estudio de prevalencia [5]. Grimes y Schulz ubican este tipo de diseño como un estudio descriptivo junto con el reporte de caso, serie de casos y los estudios de vigilancia epidemiológica [6]. No obstante, cuando el estudio incluye un grupo de comparación o grupo control, estos autores lo identifican como un diseño observacional analítico [7]; en este caso, el propósito se centra en la búsqueda de factores asociados con un evento y en la generación de hipótesis que propicien la definición de variables de riesgo, con diseños epidemiológicos más complejos como los estudios de casos y controles, y los estudios de cohortes [8].
Los primeros estudian personas que presentan el evento de interés y las comparan con aquellas que no lo presentan. Generalmente, este tipo de diseño no requiere una alta inversión en dinero y los resultados pueden obtenerse en periodos cortos de tiempo [9]. De otro lado, los estudios de cohortes se refieren al seguimiento (retrospectivo o prospectivo) que se realiza a dos o más grupos de participantes expuestos y no expuestos a la espera de la aparición del evento de interés [10].

Es indispensable conocer en detalle cada uno de los diseños epidemiológicos para elegir el tipo de estudio más adecuado según la dinámica del proceso investigativo y las variables o características que pueden ser recolectadas, con el propósito de capturar el dato de la manera más válida y confiable posible.

Por otra parte, el trabajo de grado se define como "un estudio crítico-científico, con amplitud suficiente para plantear un tema, analizarlo y sacar conclusiones objetivas y fundadas, sobre el tema propuesto" de acuerdo con el Título 8 Artículo 26 del Reglamento Particular de la Facultad de Odontología de la Universidad Santo Tomás [11]. Este se exige como requisito de grado en los programas de pregrado y posgrado de la Facultad por lo que su ejecución es de obligatorio cumplimiento para obtener el título de profesional, especialista o magíster según el caso.

Por tal motivo, cada programa contempla un número de créditos para los espacios académicos que forman a los estudiantes en investigación: en pregrado se ofrecen tres espacios académicos con un total de seis créditos, la Especialización en Endodoncia y en Rehabilitacion Oral cuenta con ocho créditos cada una, la Especialización en Ortodoncia tiene diez al igual que la Especialización en Periodoncia, y la Maestría en Odontología cuenta con 25 créditos [12-17].

El tipo de estudio epidemiológico realizado en cada uno de los trabajos de grado se relaciona con la temática por desarrollar, la viabilidad del proyecto y la finalidad de cada uno de estos espacios académicos, entre otros. Al día de hoy, no existe un estudio que caracterice los aspectos más relevantes de los trabajos de grado de la Facultad de Odontología de la Universidad Santo Tomás, si se tiene en cuenta que la formación en investigación en pregrado y posgrado está contemplada en la Línea 3 (Proyección social e investigación perti- 
nentes) del Plan Integral Multicampus 2016-2027 [18]. Así mismo, este estudio permite fortalecer el proceso de investigación a partir de los espacios académicos en los que se forma a los estudiantes.

De esta manera, el objetivo de este trabajo fue determinar el diseño de estudio epidemiológico más frecuente y la dinámica de producción científica en los trabajos de grado presentados como requisito de grado de los programas de pregrado y posgrado de la Facultad de Odontología de la Universidad Santo Tomás Seccional Bucaramanga, cuyo resumen se encuentra publicado en el Repositorio Institucional desde el 2014.

\section{MATERIALES Y MÉTODOS}

Se realizó un estudio observacional descriptivo de corte transversal con la población total de trabajos de grado vinculados con la Facultad de Odontología a enero 10 de 2021 y publicados en la colección "Tesis, Trabajos de Grado y Disertaciones Académicas" incluida en el Repositorio Institucional del Centro de Recursos para el Aprendizaje y la Investigación (CRAI) de la Universidad Santo Tomás Seccional Bucaramanga. Esta población estaba constituida por 341 trabajos de grado vinculados con el programa de Odontología, 54 pertenecían al programa de Especialización en Endodoncia de Bogotá y Bucaramanga, 34 correspondían al programa de Especialización en Ortodoncia, 21 al de Especialización en Periodoncia, 23 al de Especialización en Rehabilitación Oral y, seis trabajos pertenecían a la Maestría en Odontología.

Debido a que se evaluaron los 479 títulos no se calculó un tamaño de muestra y tampoco se realizó un muestreo. Se incluyeron todos los títulos de los trabajos de grado a partir de 2014 que no se encontraran repetidos. Las variables se obtuvieron a partir del "registro completo del ítem" de cada uno de los trabajos evaluados dado que algunos de ellos no estaban disponibles. De esta manera, se analizó la disponibilidad del trabajo para ser leído en texto completo, el número de estudiantes autores del trabajo, el nombre del director y su nivel de escolaridad, el número de codirectores, el nombre del primer codirector en caso de que hubiese más de uno, su nivel de escolaridad, el año de publicación del trabajo en el repositorio, el programa al que correspondía y el tipo de estudio según lo reportado en el resumen al que se podía acceder en el sitio web mencionado.
Adicionalmente, se evalúo la divulgación del trabajo en una revista científica, para lo cual se consultó el índice de contenidos de la Revista Ustasalud, las publicaciones de los miembros de los grupos de investigación avalados por la Facultad de Odontología de la Universidad Santo Tomás y, además, se realizó la búsqueda del título del trabajo y del nombre del estudiante en el motor de búsqueda web Google [19]. En caso de que se encontrara una publicación con un título diferente al del trabajo de grado, pero que aparecieran como autores los nombres de los estudiantes y directores, se contactó al director para verificar que el estudio publicado correspondia al trabajo de grado. Por último, se identificó un término DeCS y un término MeSH de acuerdo con el título del trabajo, estos fueron consultados en la página en línea de la Biblioteca Virtual en Salud (https://decs.bvsalud.org/) debido a que algunas de las palabras clave proporcionadas en la información del trabajo no cumplían con esta exigencia.

\section{Procedimiento}

El trabajo de campo se realizó entre el 2 de diciembre de 2020 al 10 de enero de 2021, para lo cual los investigadores acordaron la forma cómo se reuniría la información que fue registrada en una base de Microsoft Excel creada para tal fin. Uno de los investigadores recogió los datos según lo acordado y si existía alguna inquietud esta se resolvía en consenso.

Después de recoger los datos, se hizo un control de calidad de la información con el $20 \%$ de los títulos de los trabajos de grado, es decir, se revisaron 96 observaciones seleccionadas al azar a través de la generación de números aleatorios sin repetición en el sitio web http://www.alazar.info/generador-de-numeros-aleatorios-sin-repeticion. Se encontró que tres $(3,1 \%)$ observaciones contenían un error cada una.

\section{Análisis estadístico}

Se obtuvieron frecuencias y proporciones para las variables cualitativas y medidas de tendencia central y dispersión para las cuantitativas, se utilizó el software estadístico Stata I/C versión 14. Además, se generó una nube de palabras con la frecuencia de los términos DeCS y MeSH en el sitio web cuya dirección electrónica es https://bit.ly/38mwvCN. 


\section{Consideraciones éticas}

Según el Capítulo I Artículo 11 de la Resolución 08430 de 1993, este estudio se consideró como una "investigación sin riesgo", porque utilizó herramientas documentales [20]; la información analizada es de libre acceso y público conocimiento.

Así mismo, el Artículo 31 de la Ley 23 de 1982 (Protección del Derecho de Autor en Colombia) menciona que:

"Es permitido citar a un autor transcribiendo los pasajes necesarios, siempre que éstos no sean tantos y seguidos que razonadamente puedan considerarse como una reproducción simulada y sustancial, que redunde en perjuicio del autor de la obra de donde se toman. En cada cita deberá mencionarse el nombre del autor de la obra citada y el título de dicha obra" [21].

Sin embargo, este trabajo no cita apartados de los trabajos revisados y solo refiere algunos nombres de directores y codirectores, información pública reportada en el Repositorio Institucional (https://repository.usta. edu.co/handle/11634/91).

\section{RESULTADOS}

Se analizaron los datos de los 479 trabajos de grado publicados hasta enero 10 de 2021. De estos, dos se encontraban repetidos y uno, tenía fecha anterior a 2014; los tres correspondían al programa de Odontología. De esta manera, se evaluó la información correspondiente a 476 trabajos: $338(71,0 \%)$ pertenecían a pregrado y 138 (29,0\%) estaban vinculados con los programas de posgrado. La Especialización en Endodoncia presentó el mayor número al tener $54(11,3 \%)$ trabajos, seguido por la Especialización en Ortodoncia con $34(7,1 \%)$, la Especialización en Rehabilitación Oral con 23 (4,8\%), la Especialización en Periodoncia con $21(4,4 \%)$ y, por último, la Maestría en Odontología con seis $(1,3 \%)$ trabajos de grado.

De los 476 trabajos de grado, 360 (75,6\%) se encontraban disponibles en texto completo. El promedio de estudiantes por trabajo fue 3,0 $\pm 1,1$ (mediana: 3,0 RIQ: 2 - 4) con un número mínimo de un estudiante y un máximo de seis. La Tabla 1 muestra el número de estudiantes autores de los trabajos de grado según el programa en el que estaban matriculados; cabe resaltar que no se presentan los datos de los seis trabajos de la Maestría en Odontología, porque este programa exige que el trabajo de grado se realice de forma individual.

Tabla 1. Medidas de tendencia central y dispersión relacionadas con el número de estudiantes autores de los trabajos de grado según el programa en el que estaban matriculados

\begin{tabular}{lccc}
\hline \multicolumn{1}{c}{ Variable } & $\mathbf{x} \pm \mathrm{DE}$ & $\mathrm{Me}(\mathrm{RIQ})$ & Mín - Max \\
\hline Programa & & & \\
Odontología & $3,1 \pm 1,1$ & $3(2-4)$ & $1-6$ \\
E. en Endodoncia & $2,7 \pm 0,7$ & $3(2-3)$ & $1-4$ \\
E. en Ortodoncia & $2,8 \pm 1,0$ & $3(2-4)$ & $1-4$ \\
E. en Periodoncia & $2,6 \pm 0,9$ & $2(2-3)$ & $1-5$ \\
$\begin{array}{l}\text { E. en Rehabilitación } \\
\text { Oral }\end{array}$ & $2,9 \pm 0,9$ & $3(2-3)$ & $1-4$ \\
\hline
\end{tabular}

$E$ : Especialización. $\mathrm{x} \pm \mathrm{DE}$ : promedio \pm desviación estándar. Me (RIQ): Mediana (Rango Intercuartílico). Min - Max: mínimo - máximo.

Ciento nueve docentes fueron los responsables de dirigir los 476 trabajos de grado analizados en este estudio, quince de ellos dirigieron el $50 \%$ de estos; de otra parte, $173(36,3 \%)$ trabajos de grado reportaron uno a tres codirectores. Al evaluar la dirección de trabajos de grado de posgrado, se encontró que 62 (44,9\%) docentes dirigieron uno o más trabajos de grado, y al revisar la información sobre codirecciones se observó que 61 (44,2\%) trabajos de grado tenían uno a dos codirectores.

En la Tabla 2 se observa el nivel de escolaridad de directores, primer codirector en caso de que se presentara más de uno y el año de publicación del trabajo de grado en el Repositorio Institucional. Se encontró que el grado de escolaridad del director de $186(39,1 \%)$ trabajos corresponde a magíster; de estos, 159 (47,0\%) estaban vinculados con el programa de Odontología. Así mismo, se evidenció una mayor frecuencia del nivel de escolaridad magíster en los codirectores al encontrar que $58(33,5 \%)$ trabajos de grado así lo reportaban.

Con respecto al año de publicación del trabajo de grado en el Repositorio Institucional, se observó que 2018 fue el año con mayor publicación de trabajos vinculados con los programas de Odontología y Especialización en Endodoncia con 73 (21,6\%) y 18 (33,3\%), respectivamente (Tabla 2 ). 
Tabla 2. Nivel de escolaridad del director, primer codirector y año de publicación del trabajo de grado en la Colección de la Facultad de Odontología del Repositorio Institucional de la Universidad Santo Tomás desde 2014

\begin{tabular}{|c|c|c|c|c|c|c|c|}
\hline Variable & $\begin{array}{c}\text { Global } \\
\text { n (\%) }\end{array}$ & $\begin{array}{c}\text { Odont. } \\
\text { n (\%) }\end{array}$ & $\begin{array}{c}\text { Endod. } \\
\text { n (\%) }\end{array}$ & $\begin{array}{c}\text { Ortod. } \\
\text { n (\%) }\end{array}$ & $\begin{array}{c}\text { Period. } \\
\text { n (\%) }\end{array}$ & $\begin{array}{c}\text { Rehab. } \\
\text { n (\%) }\end{array}$ & $\begin{array}{c}\text { Maest. } \\
\text { n (\%) }\end{array}$ \\
\hline \multicolumn{8}{|l|}{ Esc. del director } \\
\hline Profesional & $9(1,9)$ & $9(2,7)$ & $0(0,0)$ & $0(0,0)$ & $0(0,0)$ & $0(0,0)$ & $0(0,0)$ \\
\hline Especialista & $74(15,6)$ & $71(21,0)$ & $0(0,0)$ & $2(5,9)$ & $1(4,8)$ & $0(0,0)$ & $0(0,0)$ \\
\hline Especialista clínico & $167(35,1)$ & $77(22,8)$ & $43(79,6)$ & $18(52,9)$ & $15(71,4)$ & $14(60,9)$ & $0(0,0)$ \\
\hline Magíster & $186(39,1)$ & $159(47,0)$ & $8(14,8)$ & $12(35,3)$ & $3(14,3)$ & $2(8,7)$ & $2(33,3)$ \\
\hline Doctorado & $34(7,1)$ & $22(6,5)$ & $3(5,6)$ & $2(5,9)$ & $2(9,5)$ & $1(4,4)$ & $4(66,7)$ \\
\hline Otro & $6(1,3)$ & $0(0,0)$ & $0(0,0)$ & $0(0,0)$ & $0(0,0)$ & $6(26,1)$ & $0(0,0)$ \\
\hline \multicolumn{8}{|l|}{ Esc. del codirector } \\
\hline Profesional & $10(5,8)$ & $10(8,9)$ & $0(0,0)$ & $0(0,0)$ & $0(0,0)$ & $0(0,0)$ & $0(0,0)$ \\
\hline Especialista & $28(16,2)$ & $23(20,5)$ & $3(9,1)$ & $1(10,0)$ & $1(11,1)$ & $0(0,0)$ & $0(0,0)$ \\
\hline Especialista clínico & $52(30,1)$ & $16(14,3)$ & $23(69,7)$ & $4(40,0)$ & $5(55,6)$ & $4(57,1)$ & $0(0,0)$ \\
\hline Magíster & $58(33,5)$ & $46(41,1)$ & $6(18,2)$ & $3(30,0)$ & $2(22,2)$ & $1(14,3)$ & $0(0,0)$ \\
\hline Doctorado & $20(11,6)$ & $15(13,4)$ & $0(0,0)$ & $2(20,0)$ & $1(11,1)$ & $0(0,0)$ & $2(100,0)$ \\
\hline Otro & $5(2,9)$ & $2(1,8)$ & $1(3,0)$ & $0(0,0)$ & $0(0,0)$ & $2(28,6)$ & $0(0,0)$ \\
\hline \multicolumn{8}{|l|}{ Año de publicación } \\
\hline 2014 & $23(4,8)$ & $19(5,6)$ & $0(0,0)$ & $3(8,8)$ & $0(0,0)$ & $1(4,4)$ & $0(0,0)$ \\
\hline 2015 & $67(14,1)$ & $48(14,2)$ & $3(5,6)$ & $7(20,6)$ & $5(23,8)$ & $4(17,4)$ & $0(0,0)$ \\
\hline 2016 & $81(17,0)$ & $57(16,9)$ & $10(18,5)$ & $8(23,5)$ & $3(14,3)$ & $3(13,0)$ & $0(0,0)$ \\
\hline 2017 & $68(14,3)$ & $48(14,2)$ & $6(11,1)$ & $5(14,7)$ & $4(19,1)$ & $5(21,7)$ & $0(0,0)$ \\
\hline 2018 & $102(21,4)$ & $73(21,6)$ & $18(33,3)$ & $3(8,8)$ & $4(19,1)$ & $3(13,0)$ & $1(16,7)$ \\
\hline 2019 & $65(13,7)$ & $39(11,5)$ & $11(20,4)$ & $3(8,8)$ & $3(14,3)$ & $4(17,4)$ & $5(83,3)$ \\
\hline 2020 & $70(14,7)$ & $54(16,0)$ & $6(11,1)$ & $5(14,7)$ & $2(9,5)$ & $3(13,0)$ & $0(0,0)$ \\
\hline
\end{tabular}

Esc.: Escolaridad. Odont.: Odontología. Endod.: Especialización en Endodoncia. Ortod.: Especialización en Ortodoncia. Period.: Especialización en Periodoncia. Rehab.: Especialización en Rehabilitación Oral. Maest.: Maestría en Odontología.

En relación con el tipo de estudio, más de la mitad de los trabajos de grado correspondian a estudios observacionales ( $n=252,52,9 \%)$, siendo el tipo de estudio más frecuente en cuatro de los seis programas académicos analizados. Al revisar cuál de los diseños observacionales era el más utilizado, se evidenció que más del $80 \%$ de los trabajos de grado fue de corte transversal. De los 91 $(19,1 \%)$ estudios experimentales, $55(60,4 \%)$ correspondían a estudios In vitro y de estos, 21 (38,2\%) se realizaron en la Especialización en Endodoncia (Tabla 3).

En cuanto a la divulgación de los trabajos de grado en revistas científicas, se encontraron 13 (2,7\%) publicaciones en nueve revistas científicas, seis de ellas editadas en Colombia. En la Tabla 4 se evidencia que 9 $(69,2 \%)$ artículos pertenecían al programa de Odonto- logía, $2(15,4 \%)$ a la Especialización en Endodoncia, 1 $(7,7 \%)$ a la Especialización en Ortodoncia, y 1 (7,7\%) al programa de Maestría en Odontología. El orden de aparición de los autores inició con los estudiantes en 12 $(92,3 \%)$ artículos y $6(66,7 \%)$ directores citados como autores eran miembros de los grupos de investigación avalados por la Universidad Santo Tomás. Cabe señalar que de los $116(24,4 \%)$ trabajos de grado que no se encontraban disponibles en texto completo, solo $4(3,4 \%)$ se han publicado en una revista científica.

Las figuras 1 y 2 muestran las nubes de términos DeCS y MeSH. El término DeCS y MeSH más frecuente fue estudiantes (students) con $21(4,4 \%)$ observaciones seguida de salud bucal (oral health) con $16(3,4 \%)$ y endodoncia (endodontics) con 14 (2,9\%). 
Tabla 3. Diseños de estudios epidemiológicos utilizados en los trabajos de grado publicados en la Colección de la Facultad de Odontología del Repositorio Institucional de la Universidad Santo Tomás desde 2014

\begin{tabular}{|c|c|c|c|c|c|c|c|}
\hline Variable & $\begin{array}{c}\text { Global } \\
\text { n (\%) }\end{array}$ & $\begin{array}{c}\text { Odont. } \\
\text { n (\%) }\end{array}$ & $\begin{array}{c}\text { Endod. } \\
\text { n (\%) }\end{array}$ & $\begin{array}{l}\text { Ortod. } \\
\text { n (\%) }\end{array}$ & $\begin{array}{c}\text { Period. } \\
\text { n (\%) }\end{array}$ & $\begin{array}{c}\text { Rehab. } \\
\text { n (\%) }\end{array}$ & $\begin{array}{c}\text { Maest. } \\
\text { n (\%) }\end{array}$ \\
\hline \multicolumn{8}{|l|}{ Diseño del estudio } \\
\hline Observacional & $252(52,9)$ & $201(59,5)$ & $21(38,9)$ & $14(41,2)$ & $8(38,1)$ & $4(17,4)$ & $4(66,7)$ \\
\hline Experimental & $91(19,1)$ & $37(11,0)$ & $24(44,4)$ & $9(26,5)$ & $2(9,5)$ & $17(73,9)$ & $2(33,3)$ \\
\hline Ev. Tecnología Diagnóstica & $26(5,5)$ & $14(4,1)$ & $0(0,0)$ & $7(20,6)$ & $5(23,8)$ & $0(0,0)$ & $0(0,0)$ \\
\hline Revisión & $101(21,2)$ & $85(25,2)$ & $7(13,0)$ & $1(2,9)$ & $6(28,6)$ & $2(8,7)$ & $0(0,0)$ \\
\hline Longitudinal & $4(0,8)$ & $1(0,3)$ & $0(0,0)$ & $3(0,8)$ & $0(0,0)$ & $0(0,0)$ & $0(0,0)$ \\
\hline Otro & $2(0,4)$ & $0(0,0)$ & $2(3,7)$ & $0(0,0)$ & $0(0,0)$ & $0(0,0)$ & $0(0,0)$ \\
\hline \multicolumn{8}{|l|}{ Estudios observacionales } \\
\hline Reporte /serie de caso & $8(3,2)$ & $4(2,0)$ & $4(19,1)$ & $0(0,0)$ & $0(0,0)$ & $0(0,0)$ & $0(0,0)$ \\
\hline Transversal & $237(94,1)$ & $192(95,5)$ & $17(81,0)$ & $12(85,7)$ & $8(100,0)$ & $4(100,0)$ & $4(100,0)$ \\
\hline Casos y controles & $7(2,8)$ & $5(2,5)$ & $0(0,0)$ & $2(14,3)$ & $0(0,0)$ & $0(0,0)$ & $0(0,0)$ \\
\hline \multicolumn{8}{|l|}{ Estudios experimentales } \\
\hline Ensayo aleatorizado & $9(9,9)$ & $6(16,2)$ & $1(4,2)$ & $0(0,0)$ & $0(0,0)$ & $2(11,8)$ & $0(0,0)$ \\
\hline In vitro & $55(60,4)$ & $10(27,0)$ & $21(87,6)$ & $8(88,9)$ & $0(0,0)$ & $14(82,4)$ & $2(100,0)$ \\
\hline Cuasi-experimental & $24(26,4)$ & $19(51,4)$ & $1(4,2)$ & $1(11,1)$ & $2(100,0)$ & $1(5,9)$ & $0(0,0)$ \\
\hline Ex vivo & $3(3,3)$ & $2(5,4)$ & $1(4,2)$ & $0(0,0)$ & $0(0,0)$ & $0(0,0)$ & $0(0,0)$ \\
\hline \multicolumn{8}{|l|}{ Ev. Tecnología Diagnóstica } \\
\hline Estudios de validación & $11(42,3)$ & $6(42,9)$ & $0(0,0)$ & $2(28,6)$ & $3(60,0)$ & $0(0,0)$ & $0(0,0)$ \\
\hline Estudios de reproducibilidad & $13(50,0)$ & $7(50,0)$ & $0(0,0)$ & $4(57,1)$ & $2(40,0)$ & $0(0,0)$ & $0(0,0)$ \\
\hline Traducción transcultural & $2(7,7)$ & $1(7,1)$ & $0(0,0)$ & $1(14,3)$ & $0(0,0)$ & $0(0,0)$ & $0(0,0)$ \\
\hline \multicolumn{8}{|l|}{ Estudios de revisión } \\
\hline Literatura & $13(12,9)$ & $11(12,9)$ & $1(14,3)$ & $0(0,0)$ & $0(0,0)$ & $1(50,0)$ & $0(0,0)$ \\
\hline Sistemática & $55(54,5)$ & $45(52,9)$ & $3(42,9)$ & $1(100,0)$ & $5(83,3)$ & $1(50,0)$ & $0(0,0)$ \\
\hline Bibliométrica & $30(29,7)$ & $27(31,8)$ & $2(28,6)$ & $0(0,0)$ & $1(16,7)$ & $0(0,0)$ & $0(0,0)$ \\
\hline "Scoping review" & $2(2,0)$ & $1(1,2)$ & $1(14,3)$ & $0(0,0)$ & $0(0,0)$ & $0(0,0)$ & $0(0,0)$ \\
\hline "Umbrella review" & $1(1,0)$ & $1(1,2)$ & $0(0,0)$ & $0(0,0)$ & $0(0,0)$ & $0(0,0)$ & $0(0,0)$ \\
\hline
\end{tabular}

Odont.: Odontología. Endod.: Especialización en Endodoncia. Ortod.: Especialización en Ortodoncia. Period.: Especialización en Periodoncia. Rehab.: Especialización en Rehabilitación Oral. Maest.: Maestría en Odontología.

Tabla 4. Frecuencia de divulgación de los trabajos de grado de los programas académicos en revistas científicas según la citación del nombre del director y el nombre abreviado de la publicación

\begin{tabular}{lccccccc}
\hline \multicolumn{1}{c}{ Variable } & $\begin{array}{c}\text { Global } \\
\mathbf{n}(\%)\end{array}$ & $\begin{array}{c}\text { Odont. } \\
\mathbf{n}(\%)\end{array}$ & $\begin{array}{c}\text { Endod. } \\
\mathbf{n}(\%)\end{array}$ & $\begin{array}{c}\text { Ortod. } \\
\mathbf{n}(\%)\end{array}$ & $\begin{array}{c}\text { Period. } \\
\mathbf{n}(\%)\end{array}$ & $\begin{array}{c}\text { Rehab. } \\
\mathbf{n}(\%)\end{array}$ & $\begin{array}{c}\text { Maest. } \\
\mathbf{n}(\%)\end{array}$ \\
\hline $\begin{array}{l}\text { Nombre del director } \\
\text { Álvarez-Martínez JJ }\end{array}$ & $1(7,7)$ & $1(11,1)$ & $0(0,0)$ & $0(0,0)$ & $0(0,0)$ & $0(0,0)$ & $0(0,0)$ \\
Amaya-Bautista C & $1(7,7)$ & $1(11,1)$ & $0(0,0)$ & $0(0,0)$ & $0(0,0)$ & $0(0,0)$ & $0(0,0)$ \\
Buitrago-Rojas SM & $1(7,7)$ & $0(0,0)$ & $1(50,0)$ & $0(0,0)$ & $0(0,0)$ & $0(0,0)$ & $0(0,0)$ \\
Castellanos-Dominguez YZ & $2(15,4)$ & $2(22,2)$ & $0(0,0)$ & $0(0,0)$ & $0(0,0)$ & $0(0,0)$ & $0(0,0)$ \\
Flórez LT & $1(7,7)$ & $1(11,1)$ & $2(0,0)$ & $0(0,0)$ & $0(0,0)$ & $0(0,0)$ & $0(0,0)$
\end{tabular}




\begin{tabular}{lccccccc}
\hline \multicolumn{1}{c}{ Variable } & $\begin{array}{c}\text { Global } \\
\mathbf{n}(\%)\end{array}$ & $\begin{array}{c}\text { Odont. } \\
\mathbf{n}(\%)\end{array}$ & $\begin{array}{c}\text { Endod. } \\
\mathbf{n}(\%)\end{array}$ & $\begin{array}{c}\text { Ortod. } \\
\mathbf{n}(\%)\end{array}$ & $\begin{array}{c}\text { Period. } \\
\mathbf{n}(\%)\end{array}$ & $\begin{array}{c}\text { Rehab. } \\
\mathbf{n}(\%)\end{array}$ & $\begin{array}{c}\text { Maest. } \\
\mathbf{n}(\%)\end{array}$ \\
\hline Mejía LP & $1(7,7)$ & $1(11,1)$ & $0(0,0)$ & $0(0,0)$ & $0(0,0)$ & $0(0,0)$ & $0(0,0)$ \\
Rincón-Méndez AY & $1(7,7)$ & $0(0,0)$ & $0(0,0)$ & $0(0,0)$ & $0(0,0)$ & $0(0,0)$ & $1(100,0)$ \\
\hline Rodríguez MJ & $2(15,4)$ & $2(22,2)$ & $0(0,0)$ & $0(0,0)$ & $0(0,0)$ & $0(0,0)$ & $0(0,0)$ \\
Rueda-Velásquez SJ & $1(7,7)$ & $0(0,0)$ & $1(50,0)$ & $0(0,0)$ & $0(0,0)$ & $0(0,0)$ & $0(0,0)$ \\
\hline Torres EA & $2(15,4)$ & $1(11,1)$ & $0(0,0)$ & $1(100,0)$ & $0(0,0)$ & $0(0,0)$ & $0(0,0)$ \\
\hline Revistas científicas & & & & & $0(0,0)$ & $0(0,0)$ & $0(0,0)$ \\
\hline Acta Odontol Colomb & $1(7,7)$ & $0(0,0)$ & $1(50,0)$ & $0(0,0)$ & $0(0)$ & $0(0,0)$ & $0(0,0)$ \\
\hline Acta Odontol Latinoam & $1(7,7)$ & $1(11,1)$ & $0(0,0)$ & $0(0,0)$ & $0(0,0)$ & $0(0,0)$ & $0(0,0)$ \\
\hline CES Odontol & $1(7,7)$ & $0(0,0)$ & $0(0,0)$ & $1(100,0)$ & $0(0,0)$ & $0(0,0)$ & $0(0,0)$ \\
\hline Colombia Forense & $1(7,7)$ & $0(0,0)$ & $1(50,0)$ & $0(0,0)$ & $0(0,0)$ & $0(0,0)$ & $0(0,0)$ \\
\hline Duazary & $1(7,7)$ & $1(11,1)$ & $0(0,0)$ & $0(0,0)$ & $0(0,0)$ & $0(0,0)$ & $0(0,0)$ \\
\hline Eur Arch Paediatr Dent & $1(7,7)$ & $1(11,1)$ & $0(0,0)$ & $0(0,0)$ & $0(0,0)$ & $0(0,0)$ & $0(0,0)$ \\
\hline J Oral Res & $1(7,7)$ & $1(11,1)$ & $0(0,0)$ & $0(0,0)$ & $0(0,0)$ & $0(0,0)$ & $1(100,0)$ \\
\hline Rev Fac Odont Univ Antioq & $2(15,4)$ & $1(11,1)$ & $0(0,0)$ & $0(0,0)$ & $0(0,0)$ & $0(0,0)$ \\
\hline Ustasalud & $4(30,8)$ & $4(44,4)$ & $0(0,0)$ & $0(0,0)$ & $0(0,0)$ & $0(0,0)$ \\
\hline
\end{tabular}

Odont.: Odontología. Endod.: Especialización en Endodoncia. Ortod.: Especialización en Ortodoncia. Period.: Especialización en Periodoncia. Rehab.: Especialización en Rehabilitación Oral. Maest.: Maestría en Odontología.

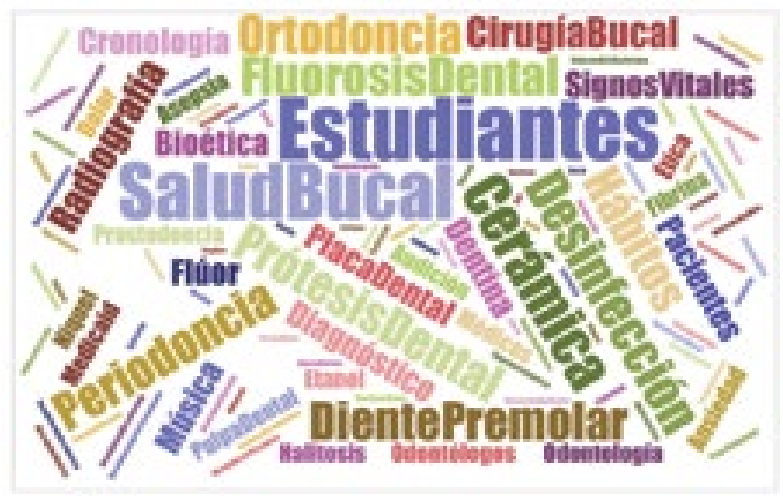

Figura 1. Nube de términos DeCS según los títulos de cada uno de los 476 trabajos de grado analizados.

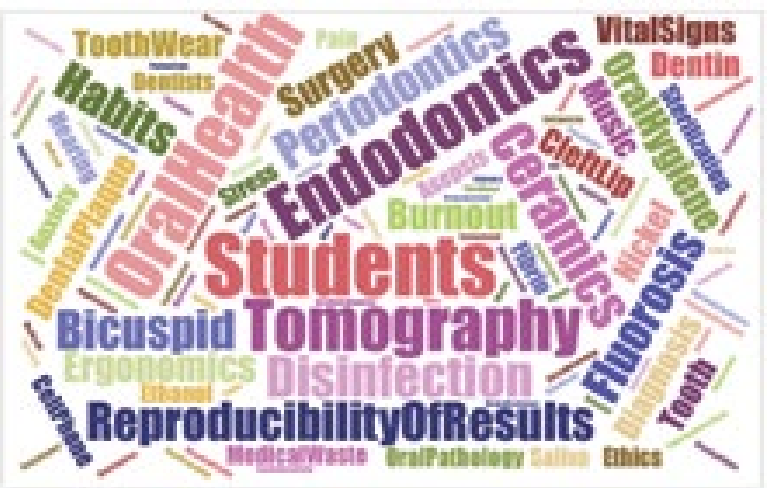

Figura 2. Nube de términos MeSH según los títulos de cada uno de los 476 trabajos de grado analizados.

\section{DISCUSIÓN}

Se encontró que el diseño de estudio epidemiológico utilizado más frecuentemente en los trabajos de grado de los programas de pregrado y posgrado de la Facultad de Odontología fue el estudio observacional de corte transversal, correspondiente en su mayoría al programa de Odontología. Este resultado coincide con otros estudios en los que se ha encontrado que el estudio observacional de corte transversal es el diseño más utilizado [22-25].

Almario-Barrera y colaboradores analizaron los resúmenes compilados en las memorias del "Encuentro Nacional de Investigación Odontológica”, organizado por la Asociación Colombiana de Facultades de Odontología (ACFO), desde el 2010 hasta el 2017, de los cuales, el 48,3\% de los 1135 resúmenes correspondía a estudios observacionales y de estos, el 40,0\% eran de corte transversal [25]. Por su parte, Rodríguez y colaboradores reportaron que 61 (57,0\%) estudios observacionales fueron publicados en la Revista Ustasalud desde el segundo semestre de 2002 hasta el primero de 2014, 50 (82,0\%) de ellos correspondian a estudios de corte transversal, en los que el primer autor tenía afiliación con la Universidad Santo Tomás como estudiante o docente [23]. 
Ahmed y colaboradores evaluaron 125 (94,7\%) trabajos de grado de estudiantes vinculados con especializaciones médico-quirúgicas que habían ejecutado estudios observacionales, $112(84,8 \%)$ eran de corte transversal [22]; sugieren que los estudiantes prefieren este tipo de estudios debido a que no se realiza seguimiento, son menos costosos y su complejidad es mediana o baja [22]. También, se ha señalado que los estudios de corte tranversal pueden obtener la aprobación ética más fácilmente, dado que los participantes no son expuestos o intervenidos [8].

A este respecto, Parker y Berman recomiendan prestar especial atención al recoger la información de los participantes y al igual que con otros diseños epidemiológicos, contar con la aprobación de un Comité de Ética, hacer el proceso de consentimiento informado y asegurar la confidencialidad de los datos [26].

Quizás la ventaja que ofrece la ejecución de estudios de corte transversal es que requieren menos tiempo. Esta situación es aprovechada por los estudiantes de pregrado y posgrado, quienes cuentan con periodos académicos limitados para desarrollar su proyecto de investigación. Así también lo declaran Grossman y Naidoo (2009) al entrevistar a estudiantes de último año de cuatro facultades de odontología en Sudáfrica, quienes reportaron que los problemas de tiempo, recursos y el trabajo en grupo obstaculizan la investigación en pregrado [27].

Navarro y colaboradores realizaron una revisión bibliométrica de los artículos publicados en revistas odontológicas incluidas en SciELO desde 2013 a 2014 y encontraron una mayor frecuencia de estudios de corte transversal ( $\mathrm{n}=121,43,2 \%)$, seguido de estudios de casos y controles $(n=68,24,3 \%)$. Los autores sugieren que la limitada publicación de estudios experimentales puede deberse a la falta de recursos o de acceso a convocatorias que los otorguen, las exigencias éticas y la presión por publicar que genera la selección de diseños epidemiológicos más sencillos de ejecutar y en los que sea más ágil la obtención de resultados [24].

En relación con el número de estudiantes que integran los trabajos de grado, se observaron discrepancias entre los diferentes programas, con excepción de la Maestría en Odontología que establece que se realice de forma individual. Cabe resaltar que el Capítulo VII
Artículo 34 Numeral 2 del Reglamento General de Posgrados Política y Lineamientos 2019 de la Universidad Santo Tomás menciona que "en las especializaciones médico-quirúrgicas y en las maestrías, podrá ser individual o en grupos de hasta tres personas, teniendo en cuenta la complejidad de la temática que se va a tratar y las condiciones propias establecidas por cada programa" [28], esta recomendación se tiene en cuenta para los programas que iniciaron en 2020 debido a que la publicación del documento fue a mediados de 2019.

Menos del 5\% de los trabajos de grado fueron publicados en revistas científicas, lo que contrasta con los hallazgos de Nieminen y colaboradores quienes encontraron que $61(23,8 \%)$ trabajos de grado de estudiantes de medicina y odontología de una Universidad en Finlandia habían sido divulgados [19]. Dada la baja frecuencia de publicación, estos autores recomiendan que desde el pregrado se incentive a los estudiantes con cursos en los que se capacite sobre el proceso de publicación, la revisión por pares, las habilidades necesarias para construir el manuscrito y la importancia de divulgar los resultados [19].

Por su parte, Salmi y colaboradores encontraron que $51(17,0 \%)$ trabajos de grado de estudiantes vinculados con 36 facultades de medicina francesas habían sido publicados en revistas científicas [29]. Resultados similares fueron obtenidos por Arriola-Quiroz y colaboradores al reportar que 85 (17,6\%) trabajos de grado de estudiantes de medicina de una facultad peruana fueron divulgados en revistas científicas, con una mayor proporción de artículos publicados en revistas de ese país ( $\mathrm{n}=68,80,0 \%)$. Al revisar el orden de autoría, los estudiantes ocuparon el primer lugar en $71(83,5 \%)$ de ellos [30].

Aunque los trabajos de grado vinculados con la Facultad de Odontología son divulgados en el Repositorio Institucional que es de libre acceso, se encontró que algunos de ellos no están disponibles en texto completo y no se han publicado en revistas científicas. Posiblemente, están ocultos porque se espera una publicación que hasta el momento no se ha realizado lo que ocasiona una importante pérdida de información.

En diferentes ámbitos se ha discutido la pertinencia de los trabajos de grado en programas clínicos de pregrado y posgrado. Si bien se ha argumentado que los recursos en dinero e infraestructura, el tiempo y 
la búsqueda de un tutor idóneo son aspectos que dificultan su ejecución, también se menciona que posiblemente es el único espacio en que el estudiante tiene un contacto muy cercano con la investigación. Kabra y Verma indican que el proceso que realiza el estudiante al identificar un tema de interés, hacer la búsqueda de literatura, formular una pregunta de investigación, plantear los objetivos, planear la recolección de la información, analizar los datos, discutir los resultados y presentar o publicar el trabajo proporciona un entrenamiento indispensable en su formación como clínico [31]. Así como lo comentan estos autores, la formación en investigación es una actividad de aprendizaje que con esfuerzo, responsabilidad y dedicación puede llegar a feliz término [31].

Como fortaleza del presente trabajo se destaca el reconocimiento de los diseños de investigación que predominan en las investigaciones desarrolladas por los estudiantes de pregrado y posgrado en la Facultad de Odontología involucrada en el estudio; de la importancia que se le otorga a estos procesos como parte integral de la formación de los estudiantes de la Facultad y de la alternativa que se abre para que los futuros profesionales puedan considerar la investigación como una opción en el marco de su ejercicio como odontólogos.

Cabe señalar que una de las limitaciones del presente trabajo fue no incluir la variable área de estudio, pues sería un aporte importante en el reconocimiento de las tendencias en la producción científica de la Facultad de Odontología de la Universidad Santo Tomás, y con este fundamento implementar las acciones que logren contribuir, tanto al área de las ciencias básicas, clínicas, como de la salud pública; esta información no era evidente en varios de los resúmenes revisados y no se consideró oportuno hacer esta clasificación desde la perspectiva de los investigadores involucrados en esta pesquisa, por los sesgos de clasificación que se podrían generar. Aspectos que sí fueron analizados por Pizarro y colaboradores, quienes evaluaron el perfil bibliométrico de las investigaciones de la Escuela de Odontología de la Universidad del Valle presentadas en los encuentros de investigación de la ACFO; los autores identificaron las características relacionadas con la temática y el área del conocimiento, la producción científica, los grupos de investigación y la importancia que tienen estos criterios en el desarrollo de la investigación en los diferentes programas [32].
Por otra parte, al sustentar el proceso investigativo a partir de la información aportada en los resúmenes, limita el alcance de los resultados y señala la necesidad de nuevas investigaciones orientadas a profundizar en aspectos metodológicos y conceptuales contenidos en los trabajos evaluados.

\section{CONCLUSIÓN}

Este trabajo encontró que los estudios observacionales de corte transversal son los más utilizados por los estudiantes vinculados con la Facultad de Odontología de la Universidad Santo Tomás, dadas sus múltiples ventajas que se ajustan a los tiempos y recursos con los que los estudiantes se aproximan a hacer un ejercicio investigativo de calidad.

\section{REFERENCIAS}

[1] Thiese MS. Observational and interventional study design types; an overview. Biochem Med (Zagreb). 2014;24(2):199-210. DOI: 10.11613/BM.2014.022

[2] Kelsey J, Whittemore A, Evans A, Thompson W. Cross Sectional and other Types of Studies. Methods in Observational Epidemiology. Second ed. New York: Oxford University Press; 1996. p. 245-67.

[3] Kesmodel US. Cross-sectional studies - what are they good for? Acta Obstet Gynecol Scand. 2018;97(4):388-93. DOI: $10.1111 /$ aogs.13331

[4] Pandis N. Cross-sectional studies. Am J Orthod Dentofacial Orthop. 2014;146(1):127-9. DOI: $10.1016 / \mathrm{j}$. ajodo.2014.05.005

[5] Rothman KJ, Lash TL. Epidemiologic Study Design with Validity and Efficiency Considerations. In: Lash TL, VanderWeele TJ, Haneuse S, Rothman KJ, editors. Modern Epidemiology. 4th ed. Philadelphia: Wolters Kluwer; 2021.

[6] Grimes DA, Schulz KF. Descriptive studies: what they can and cannot do. Lancet. 2002;359(9301):145-9. DOI: 10.1016/S0140-6736(02)07373-7

[7] Grimes DA, Schulz KF. An overview of clinical research: the lay of the land. Lancet. 2002;359(9300):57-61. DOI: 10.1016/S0140-6736(02)07283-5

[8] Wang X, Cheng Z. Cross-Sectional Studies: Strengths, Weaknesses, and Recommendations. Chest. 2020;158(1S):S65-S71. DOI: 10.1016/j.chest.2020.03.012

[9] Schulz KF, Grimes DA. Case-control studies: research in reverse. Lancet. 2002;359(9304):431-4. DOI: 10.1016/ S0140-6736(02)07605-5 
[10] Grimes DA, Schulz KF. Cohort studies: marching towards outcomes. Lancet. 2002;359(9303):341-5. DOI: 10.1016/S0140-6736(02)07500-1

[11] Universidad Santo Tomás. Reglamento Particular Facultad de Odontología. Bucaramanga: Ediciones Universidad Santo Tomás; 2017.

[12] Universidad Santo Tomás. Plan de Estudios, Pregrado Odontología: Universidad Santo Tomás; 2021 [Available from: http://facultadodontologia.ustabuca.edu.co/.

[13] Universidad Santo Tomás. Plan de Estudios, Especialización en Endodoncia: Universidad Santo Tomás; 2021 [Available from: http://facultadodontologia.ustabuca.edu.co/index.php/nuestros-programas/ specializacion-en-endodoncia.

[14] Universidad Santo Tomás. Plan de Estudios, Especialización en Rehabilitación Oral: Universidad Santo Tomás; 2021 [Available from: http://facultadodontologia.ustabuca.edu.co/index.php/nuestros-programas/ especilizacion-en-rehabilitacion-oral.

[15] Universidad Santo Tomás. Plan de Estudios, Especialización en Periodoncia: Universidad Santo Tomás; 2021 [Available from: http://facultadodontologia.ustabuca.edu.co/index.php/nuestros-programas/ especializacion-en-periodoncia.

[16] Universidad Santo Tomás. Plan de Estudios, Especialización en Ortodoncia: Universidad Santo Tomás; 2021 [Available from: http://facultadodontologia.ustabuca.edu.co/index.php/nuestros-programas/ especializacion-en-ortodoncia.

[17] Universidad Santo Tomás. Plan de Estudios, Maestría en Odontología: Universidad Santo Tomás; 2021 [Available from: http://facultadodontologia.ustabuca.edu.co/index. $\mathrm{php} /$ nuestros-programas/maestria-en-odontologia.

[18] Universidad Santo Tomás, editor. Plan Integral Multicampus PIM 2016-2027. Bogotá: Universidad Santo Tomás; 2016.

[19] Nieminen P, Sipila K, Takkinen HM, Renko M, Risteli L. Medical theses as part of the scientific training in basic medical and dental education: experiences from Finland. BMC Med Educ. 2007;7:51. DOI: 10.1186/1472-6920-7-51

[20] República de Colombia, Ministerio de Salud. Resolución No. 08430 de 4 de octubre de 1993. Bogotá 1993.

[21] Ley 23 de 1982 sobre Derechos de Autor, 1982.
[22] Ahmed Y, Kanyengo C, Akakandelwa A. Mapping Postgraduate Research at the University of Zambia: a review of dissertations for the Master of Medicine Programme. Med J Zambia. 2010;37(2):52-7.

[23] Rodríguez M, Pieruccini J, Pieruccini S, Concha-Sánchez SC. Tendencia de publicación en Ustasalud desde 2002 hasta 2014: un análisis bibliométrico. Ustasalud. 2014;13(1):40-8. DOI: 10.15332/us.v13i1.1397

[24] Navarro P, Cantín M, Ottone N. Diseños de investigación utilizados en revistas odontológicas de la red SciELO: una visión bibliométrica. Av Odontoestomatol. 2016;32(3):153-8.

[25] Almario-Barrera AJ, Concha-Sánchez SC, Angarita ÁA, Barón JV, Sampayo JP, Bueno LP, et al. Tendencias bibliométricas de los resúmenes compilados en las memorias ACFO desde 2010-2017. Ustasalud. 2019;18(1):1727. DOI: $10.15332 /$ us.v18i0.2391

[26] Parker RA, Berman NG. Cross-Sectional Studies. Planning Clinical Research. New York: Cambridge University Press; 2016.

[27] Grossman ES, Naidoo S. Attitudes towar a research component in the curriculum. J Dent Educ. 2009;73(11):1306-12.

[28] Universidad Santo Tomás. Reglamento General de Posgrados Política y Lineamientos 2019. Bogotá: Ediciones Universidad Santo Tomás; 2019.

[29] Salmi LR, Gana S, Mouillet E. Publication pattern of medical theses, France, 1993-98. Med Educ. 2001;35(1):1821. DOI: $10.1046 /$ j.1365-2923.2001.00768.x

[30] Arriola-Quiroz I, Curioso WH, Cruz-Encarnacion M, Gayoso O. Characteristics and publication patterns of theses from a Peruvian medical school. Health Info Libr J. 2010;27(2):148-54. DOI: $10.1111 /$ j.1471-1842.2010.00878.x

[31] Kabra S, Verma I. Thesis during MD: must or bust? Indian J Pediatr. 2007;74(9):864-9. DOI: $10.1007 / \mathrm{s} 12098-007-0158-\mathrm{Z}$

[32] Pizarro MA, Martínez V, Posada G, Moreno F. Investigaciones de la Escuela de Odontología de la Universidad del Valle presentadas en los encuentros de la Asociación Colombiana de Facultades de Odontología (ACFO) a 2016: Perfil bibliométrico. Rev Estomatol. 2018;26(2):219. DOI: $10.25100 /$ re.v26i2.7635

\section{Correo electrónico de los autores:}

Martha Juliana Rodríguez: marthajuro@gmail.com

Juan José Sánchez: juancho.f.t@hotmail.com

Sonia Constanza Concha-Sánchez: sococosavila@gmail.com 